Title:

Striking contiguous depigmentation across the lower limbs in Piebaldism and its Implications for Understanding Melanocytic Migration and Development.

\begin{abstract}
:
Piebaldism is a rare autosomal dominant disorder of pigmentation that is characterized by variable patches of depigmentation on the face, chest, abdomen and extremities. We describe two cases of piebaldism, where the remarkable asymmetric distribution of the depigmented patches in a connected, contiguous pattern across the legs provides embryological insights. This finding is not explained by the traditional theory that melanocytic migration only originates in the neural crest and progresses unilaterally down each leg. We propose that our cases, and other similar cases, can be explained by a recent theory of mesodermal melanocyte migration.
\end{abstract}

Introduction:

Piebaldism is a rare autosomal dominant disorder of pigmentation resulting from a KIT mutation $^{1}$ leading to a congenital reduction or absence of melanocytes in specific areas of the skin and hair. ${ }^{2}$ These areas of depigmentation are usually stable and persistent, with their severity and clinical features correlating with the site and type of mutation in the KIT gene. ${ }^{2}$ The characteristic clinical findings include variable depigmentation on the face, chest and/or abdomen, centered on the ventral midline, as well as symmetrical depigmented patches on the extremities, often involving the elbows and knees. ${ }^{3}$ A central white forelock is seen in $80-90 \%$ of patients. ${ }^{3}$ We now report two cases of piebaldism with characteristic clinical findings of piebaldism, but which were noteworthy for the striking finding of depigmented patches of the 
legs in a pattern of connected, contiguous pigment changes across the legs. We review previously reported cases demonstrating similar findings, and discuss a proposed pathogenetic basis for this observation using recent insights into developmental melanocytic patterning.

Patient One:

A 13-year-old female presented for evaluation of pigmentary anomalies present since early infancy. Specifically, she had hypopigmentation of her forehead and overlying hair, and areas of depigmentation and hypopigmentation on her lower legs and abdomen which were asymptomatic with no pain or pruritus (Figure 1). She was otherwise healthy with no medical problems. Family history revealed a history of similar findings in in both her mother and younger brother.

Physical examination demonstrated a well-demarcated area of depigmented skin on the forehead and adjacent scalp with localized white hair in the affected scalp. On her bilateral anterior and posterior lower legs two large patches of depigmented and hypopigmented skin with islands of hyperpigmentation within were noted. Remarkably, these two areas were contiguous across the legs in a continuous geometric pattern, most evident when the legs were placed in close apposition (Figure 1). Her abdomen had similar depigmented and hypopigmented macules and patches with islands of hyperpigmentation. Based on her physical exam findings, autosomal dominant family history, and a lack of sensorineural hearing loss, a clinical diagnosis of piebaldism was made. Formal genetic testing was not obtained but referral to genetics for genetic counseling and testing was recommended. Sun protection and modalities for reducing the appearance of her hypopigmented/depigmented areas were reviewed 


\section{Patient Two:}

A male infant presented at 13 months with congenital hypopigmented macular areas which were unchanged since birth. Physical exam showed hypopigmentation on the forehead in a midline triangle with no affected hair, on the abdomen from below the nipples bilaterally to below the umbilicus, and on the legs from mid-thigh to mid-calf (Figure 2). Family history was significant for the same features with or without midline anterior poliosis in the paternal grandfather and three first degree cousins. He was otherwise healthy with no history or family history of problems with vision or hearing.

\section{Discussion:}

While the clinical findings in piebaldism are well characterized, our patients' cases are noteworthy because of the evident contiguous pattern of depigmentation across both legs. Scrutiny of photographs of previously reported cases of piebaldism demonstrate this pattern, but to our knowledge, the observation that this depigmentation is contiguous across the limbs has not been commented upon. ${ }^{4,5,6}$

Contiguous pigmentary, vascular, or hamartomatous changes crossing boundaries have been previously reported in a few clinical settings. For example, similar patterns of divided nevi occur in congenital melanocytic nevi crossing the eyelids ("so-called kissing nevi") or from the glans penis to the adjacent preputial skin. It has been hypothesized that this occurs before the separation of the palpebral fissures ${ }^{7}$ and epithelial glanular placode and the epithelial preputial placode ${ }^{8}$ respectively. Weitz et al. reported a case series of "biker-glove hemangiomas" where acral infantile hemangiomas involved the fingers of hands in direct contiguity across the fingers, sparing the fingertips. They hypothesized that this pattern could be explained by a developmental 
error occurring after the finger rays are formed but before the interweb spaces are completely developed and the distal digits have differentiated. ${ }^{9}$ A similar finding has been reported with epidermal nevi. ${ }^{10}$ Such an explanation cannot be invoked for the legs, however, since the embryology of the legs supports the concept that the legs form separately as outpouchings on either side of the ventrolateral body wall and then elongate, rather than being fused with subsequent separation. ${ }^{11}$

The long-standing dogma of melanocytic development and migration is that melanocytes are derived from neural crest cells, which originate in the neural tube and then migrate, proliferate and differentiate into various tissues throughout the body after formation of the neural crest (recently reviewed in Mort, 2015). ${ }^{12}$ In this paradigm, melanocyte precursors formed in the neural crest migrate in a dorsal to ventral fashion. Melanocytes first appear in the epidermis at about week 6 of human embryonic development. While this pattern fits well with the patterns of certain congenital pigmentary abnormalities, particularly those following the lines of Blaschko, it does not explain other anomalies including large congenital melanocytic nevi nor the contiguous pigmentary patterning observed in our patients with piebaldism.

Recently Kinsler and Larue have proposed that a novel population of melanocyte precursors develops within the mesoderm at around the time of gastrulation (15 days in the human), and diffuses centrifugally from midline structures in a bilateral manner. ${ }^{13}$ The timing of this would occur before the formation of the limb buds. ${ }^{13}$ Their report provides striking clinical examples to support this hypothesis including cases with direct contiguity of giant congenital melanocytic nevi across the legs. It also includes photographs of one patient with piebaldism with findings similar to our patients. Our cases and those previously documented in the literature support the concept that piebaldism is a result of altered mesodermal melanocyte migration, 
lending further support to the concept of mesodermal melanocytic migration as a new origin of melanocyte precursor migration in humans.

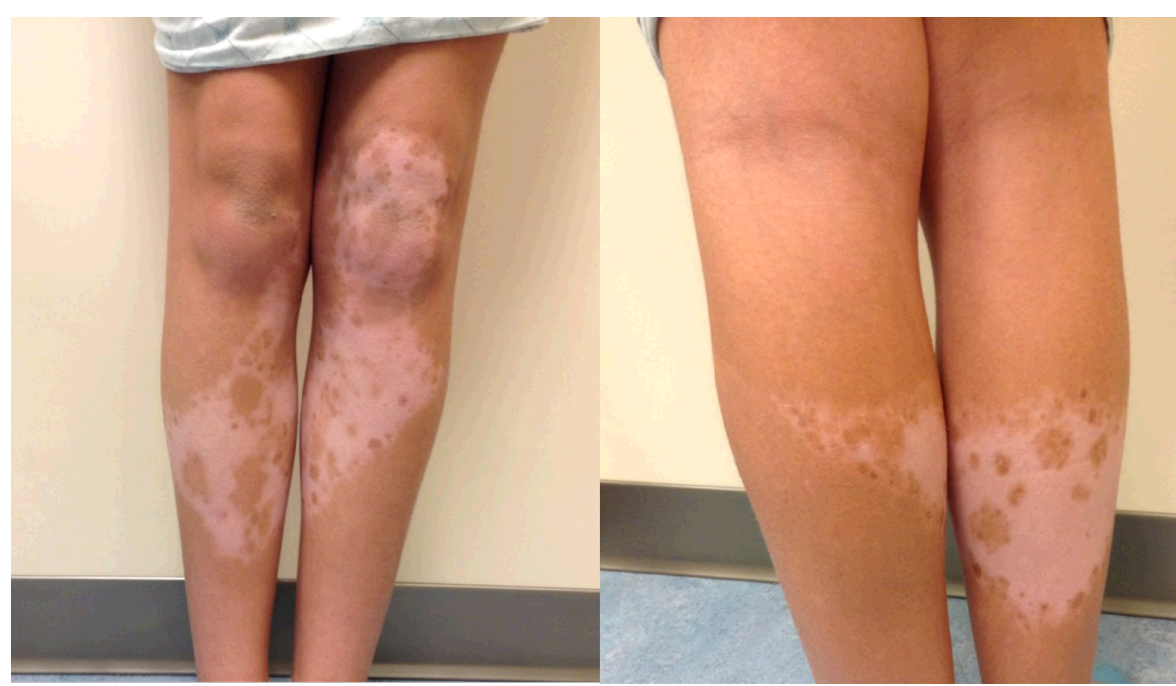

Figure 1. Patient One clinical images. Patches of depigmented and hypopigmented skin with islands of hyperpigmentation on the lower legs forming a continuous geometric pattern when the legs are placed in close apposition.

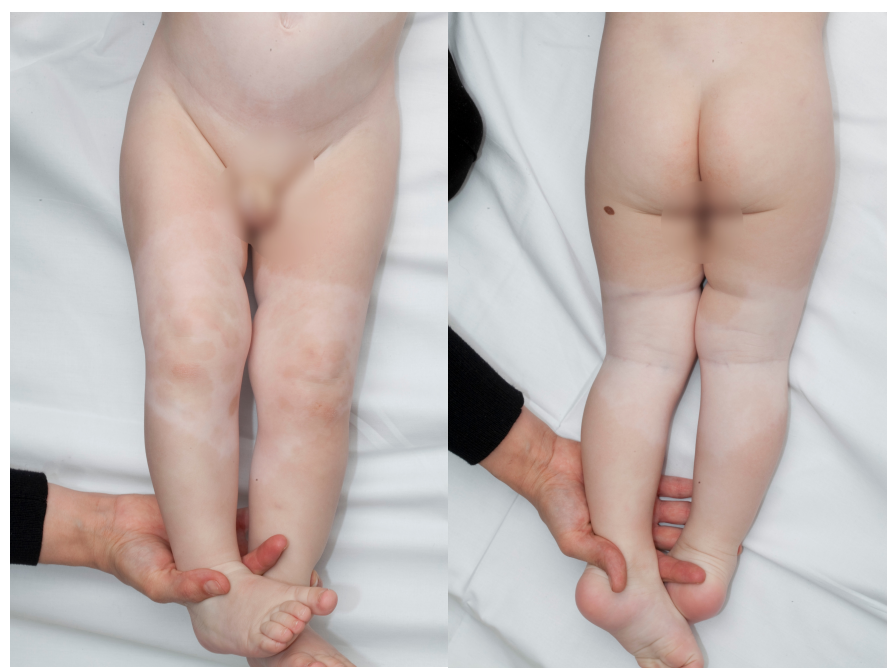

Figure 2. Patient Two clinical images showing similar findings to Patient One (Figure 1).

References: 
1. Spritz RA, Giebel LB, Holmes SA. Dominant negative and loss of function mutations of the ckit (mast/stem cell growth factor receptor) proto-oncogene in human piebaldism. Am J Hum Genet 1992;50:261-269

2. Oiso N, Fukai K, Kawada A, Suzuki T. Piebaldism. J Dermatol 2013;40(5):330- 335.

3. Treadwell PA. Systemic conditions in children associated with pigmentary changes. Clin Dermatol 2015;33:362-367.

4. Bondanza S, Bellini M, Roversi G, Raskovic D, Maurelli R, Paionni E, Paterna P, Dellambra E, Larizza L, Guerra L. Piebald trait: implication of kit mutation on in vitro melanocyte survival and on the clinical application of cultured epidermal autografts. J Invest Dermatol 2007;127(3):676-686.

5. Agarwal S, Ojha A. Piebaldism: a brief report and review of the literature. Indian Dermatology Online Journal 2012;3(2):144-147.

6. Cunliffe T. Piebaldism. Primary Care Dermatology Society. http://www.pcds.org.uk/clinicalguidance/piebaldism1. Published May 5, 2016. Updated November 20, 2017. Accessed June 27, 2018.

7. Desai SC, Walen S, Holds JB, Branham G. Divided nevus of the eyelid: Review of embryology, pathology and treatment. Am J Otolaryngol 2013;34(3):223-229

8. Desruelles F, Lacour JP, Mantoux F, Ortonne JP. Divided Nevus of the Penis: An Unusual Location. Arch Dermatol 1998;134(7):879-880

9. Weitz NA, Bayer ML, Baselga E, Torres M, Siegel D, Drolet BA, Frieden IJ, Haggstrom AN. The "biker- glove" pattern of segmental infantile hemangiomas on the hands and feet. Journal of the American Academy of Dermatology 2014;71:542-547.

10. Hayashi N, Soma Y. A case of epidermal nevi showing a divided form on the fingers. Journal of the American Academy of Dermatology 1993;29(2):281-282.

11. Sadler TW. Limbs. In: Langman's Medical Embryology. 13 ed. Philadelphia, USA: Wolters Kluwer;2015. Accessed July 9, 2018.

12. Mort RL, Jackson IJ, Patton EE. The melanocyte lineage in development and disease. Development 2015;142(4): 620-632.

13. Kinsler VA, Larue L. The patterns of birthmarks suggest a novel population of melanocyte precursors arising around the time of gastrulation. Pigment Cell Melanoma Res 2017;31:95-109. 